\title{
DEVELOPMENT OF A FRAMEWORK FOR BALLISTIC SIMULATION
}

\author{
Anh, N. T.; Anh, N. H. \& Dat, N. T. \\ Viettel Institute of Research and Development, Plot 01, Hoalac High-Tech Industrial Park, Hanoi, \\ Vietnam \\ E-Mail: anhnt480@viettel.com.vn, anhnh114@viettel.com.vn, datnt65@ viettel.com.vn
}

\begin{abstract}
The ability to predict the trajectory of ballistics under the effect of environment is a crucial factor to guarantee the fidelity of ballistic simulator systems. Therefore, this research focuses on developing a framework for modelling and simulating the trajectory of ballistics under the complex effect of environment in reality. From the beginning, the overview of framework is given which includes the process to generate the aerodynamic databases and the models for flight dynamics such as gravitational force, Coriolis force, propulsion model and environmental model especially. A data treatment method is proposed to optimize the calculation of aero databases in which the low-fidelity and high-fidelity data are fused to obtain the most accurate data as possible while minimizing the computational resources. In addition, details of non-linear flight dynamics model are analysed and make a clarification in the dynamic model section. The environmental model is also mentioned and presented in this section. Finally, several simulation results are shown to validate and verify the mathematical models of framework for ballistic simulation and then demonstrate the efficiency in calculating the trajectory with and without effect of the environmental model.

(Received in May 2018, accepted in September 2018. This paper was with the authors 1 week for 1 revision.)
\end{abstract}

Key Words: Trajectory, Non-linear Dynamics, Ballistic Simulation, Environment Effect, MultiFidelity Analysis, Flight Simulation

\section{INTRODUCTION}

The modelling and simulation of flight objects play an important role in many areas such as civil pilot training, military training and research, design of a new weapon. Typically, the response of flight simulator has been developed closer truly to reality thanks to the close collaboration between aircraft manufacturers and research communities.

However, developed platforms for modelling and simulating of full trajectory of ballistics under the effect of atmospheric disturbances are restricted. Most researches focus on separated parts of the ballistic trajectory that considers only aerodynamic model or mathematical model of flight object. Several simulation engines just provide the trajectory of bullet or bomb under limited conditions and unrealistic assumptions. In reality, the whole process to model the full trajectory of flight object is complicated and has a lot of conditions and effects need to be considered.

Carlucci and Jacobson [1] give an overview in the theory and design of the ammunition, divided into three main parts: the detail in Interior Ballistics, the difference models and the analysis of exterior ballistics is given in the second part, the impact of bullets are mentioned in the last part. However, it does not take into account the effects of turbulent winds during the moving ballistics, nor does it refer to the method of generating aerodynamic data for each ammunition. The seven-degree model is introduced in [2], applied to guided missile using Kalman filter; but, the model does not address the effects of wind. Baranowski [3] focused on different impacts of dynamic, kinematic equations and integration step on the accurate level of a projectile. The effect of wind into missile motion is considered in [4], however, it only represents a steady wind. There are a lot of conditions that use to describe the motion of projectile in [5] such as wind, Coriolis forces and Magnus forces. In these studies, only cross 
wind is considered and there is no mention to method to collect the aerodynamic coefficient. The method called "modified point mass" is established in $[6,7]$ to generate the trajectory of a projectile for a rapid result and acceptable accuracy. Though, this method only mentions the effect of steady wind in high launch angle trajectory.

In the current work, a framework for predicting the trajectory of ballistic which includes the environmental effects is proposed. Section 2 gives a brief description of the concept solving for ballistic trajectory simulation. The main motivation of section 3 is to construct identification method of the aerodynamic coefficients using both low-fidelity and highfidelity analysis. The full six degree of freedoms nonlinear equation of motion for ballistic that includes bullet and bomb which derived from Newton Second Laws is presented. Atmospheric disturbances such as wind are described in order to show how theses affect to ballistic body. The results of simulation for bomb's trajectory with alternative velocity and altitude on the disturbances of environment are calculated and compared with earlier studies. Finally, the efficiency of the developed framework is evaluated. Furthermore, this section also mentions environmental effects which are important advanced module of this framework.

\section{CONCEPT FOR BALLISTIC TRAJECTORY SIMULATION}

The detail of concept is shown in Fig. 1. At the beginning, several basic information of object is collected such as geometry, operating features. Then, aero databases (AeroDB) are generated using multi-fidelity analysis method which combines the low-fidelity analysis using empirical relations and the high-fidelity analysis using high-order method of Computational Fluid Dynamics. Dynamics model is developed in order to estimate the forces and moments which is the consequence of the interaction between the flight object and the surrounding air. The inputs of dynamics model include the wind effect, turbulence, aerodynamic table and mass parameters from configuration. Fourth-order Runge-Kutta numerical method is used to solve the ordinary differential equation and ensure the accuracy of model as well as optimize computational time. These states of ballistics are updated step by step to create the trajectory and fire table.

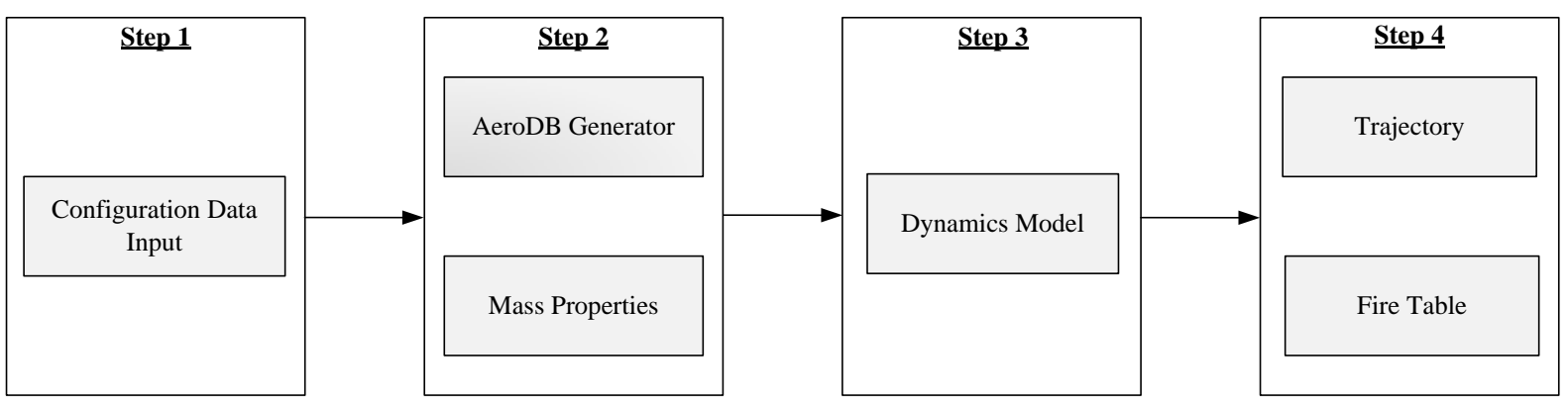

Figure 1: Conception of ballistic trajectory simulation.

\section{AERODYNAMICS PREDICTION METHOD}

Aerodynamic model for missile is derived from wind tunnel, flight testing and computational fluid dynamics (CFD). Although wind tunnel testing provides fairly accurate results, it is considered a complicated and time consuming method because several scales, case studies and measuring instruments must be taken into account during the generation of the aero databases. The most accurate method involves flight testing of actual ballistic. Unfortunately, these tests are very expensive, dangerous and time consuming to complete flight testing program for calculating aero databases that is used in full action simulation. The third method is CFD which employs a combination of data sheets, linear aerodynamic theory and empirical 
relations, low and high fidelity simulation. This method provides less reliable than the wind tunnel testing and takes CPU time particularly if estimates of coefficients are desired over a wide range of flight condition.

The ballistic trajectory strongly depends on its aerodynamic characteristics. Thus, a more accurate aerodynamic model leads to a better simulation result. In this work, as shown in Fig. 2, a multi-fidelity analysis is used in order to construct a reliable aerodynamic database which comprises six basic components as $C_{x}, C_{y}, C_{z}, C_{l}, C_{m}, C_{n}$ divided into longitudinal and lateral dynamic stability coefficients.

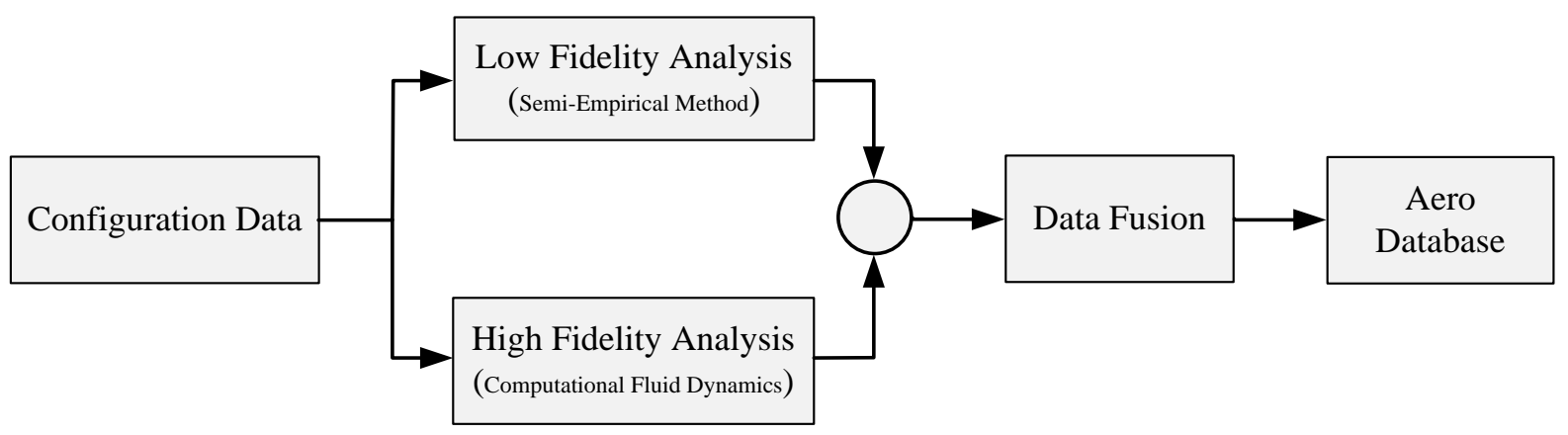

Figure 2: Aero Database Generator.

Firstly, the low-fidelity analysis is fulfilled using several software packages that allow obtaining rapid, economically and reasonably a good estimation of aerodynamic stability and control characteristics such as Athena Vortex Lattice (AVL), Missile Datcom and Digital Datcom. Missile Datcom software developed by U.S Air Force has been widely used for preliminary estimation of bombs and missile's aerodynamic coefficients and stability derivatives [8].

Secondly, the high-fidelity analysis is employs using CFD-ANSYS Fluent which uses a three-dimensional Reynolds Averaged Navier-Stokes (RANS) solution. The Spalart-Allmaras one-equation turbulence model is implemented for relatively faster estimation of high Reynolds number effect. The validation result for Fluent performed on the supersonic configurations [9]. The results show the good agreement with experiment data and numerical simulation on pressure distributions. Moreover, Fluent is one of the most popular commercial software for flow engineering. Therefore, Fluent is highly recommended for high-fidelity analysis. The mesh configuration of bomb MK82 is shown in Fig. 3, the technique of inflation is used to control $y+$ value and then exactly capture the boundary layer. Consequently, the result of high fidelity analysis is determined as the pressure distribution at two different Angles of Attack $(A o A)$ as qualitatively shown in Fig. 4 in which the dark represents for the high pressure region and contrarily.

Lastly, data fusion technique is used to combine different types of data. Here, the aim is to generate a reliable aerodynamic table for flight object with a smallest number of RANS simulation results [10-12]. The method assumes that the low-fidelity data predicts the trend of changes and the high-fidelity data are used for the absolute values. In this work, the Kriging interpolation method was used to estimate all function [13]. In addition, Co-Kriging process, based on Kriging method, is used to handle with more than one kind of data [14]. Those techniques offer a capability compared with regression methods. The interpolation goes through the sample point and it does not assume a global form of the function. In contrast to regression method, the accuracy of Kriging function always increases with an increase in the number of samples. 


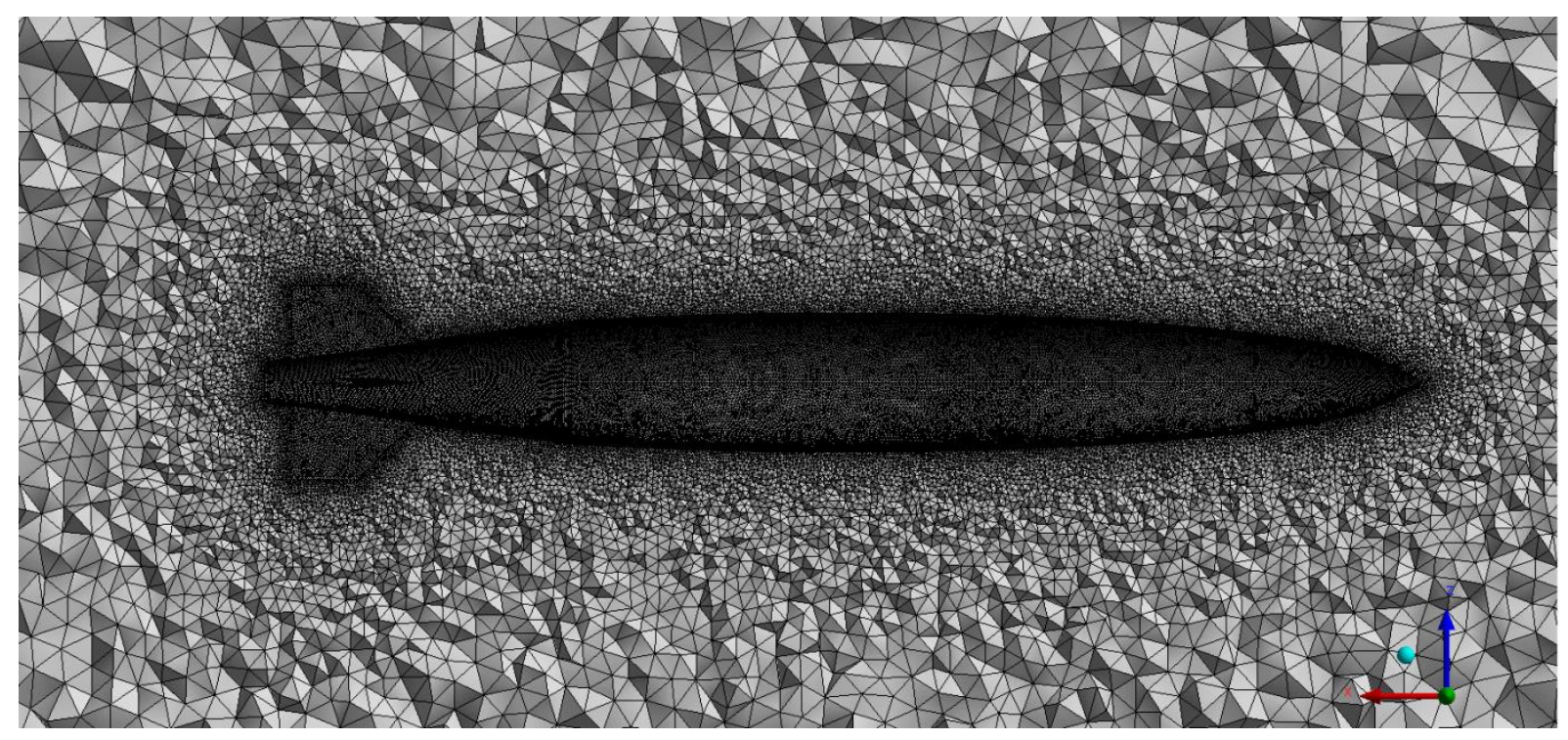

Figure 3: Unstructured mesh of MK82 bomb.

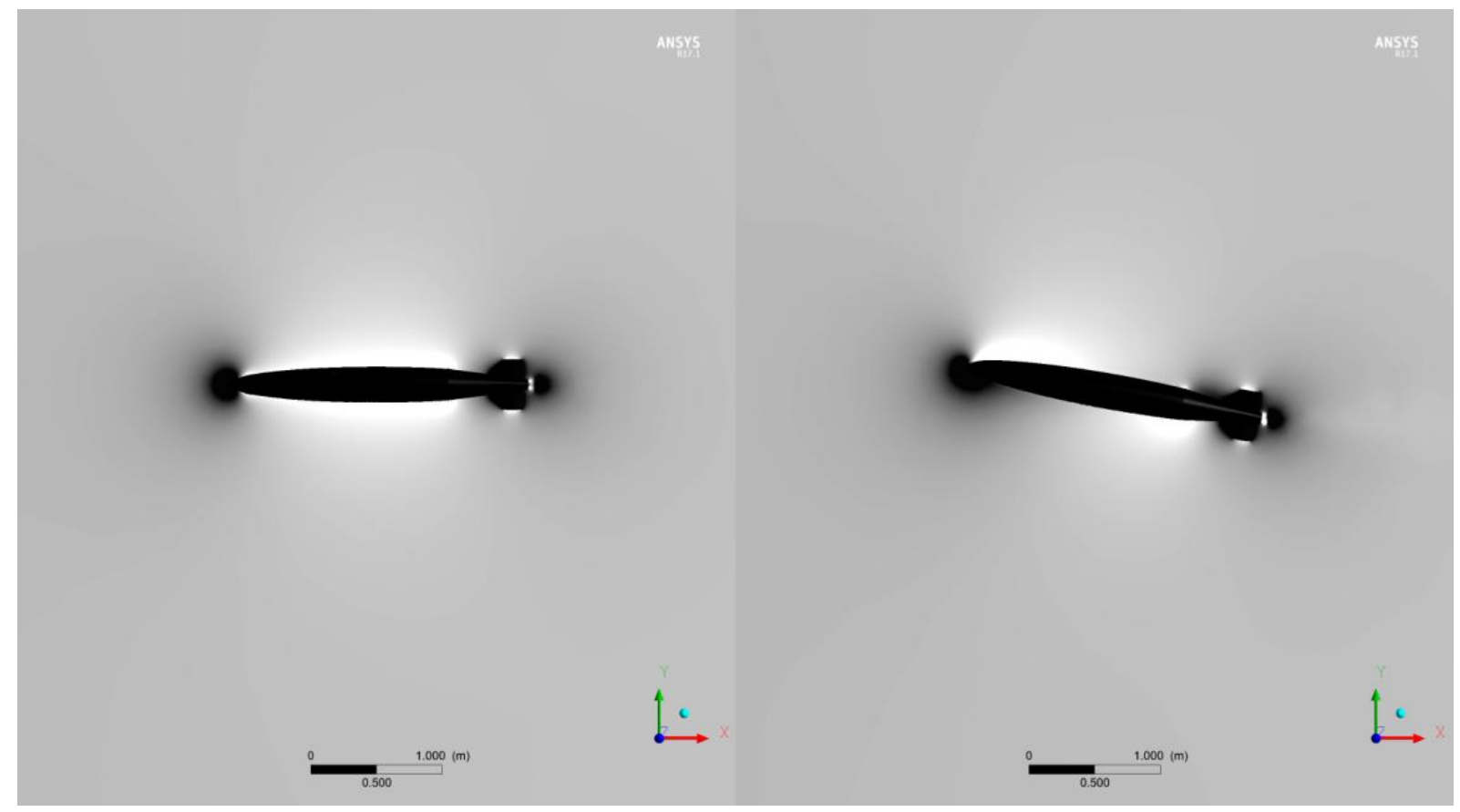

Figure 4: Pressure distribution over MK82 bomb at $A o A 0^{\circ}$ (left) and $10^{\circ}$ (right).

\section{DYNAMICS MODEL}

\subsection{Assumptions}

Following the geometry configuration and the features of bomb, unguided missile and bullet during operation, several assumptions is proposed to neglect the secondary phenomenon (aero-elastic, mass reduction) and focus on the primary factor strongly impacting to the trajectory. Concretely, the object is considered as a rigid body, constant mass and classical design with two symmetrical planes (XY and XZ). 


\subsection{Dynamic model form}

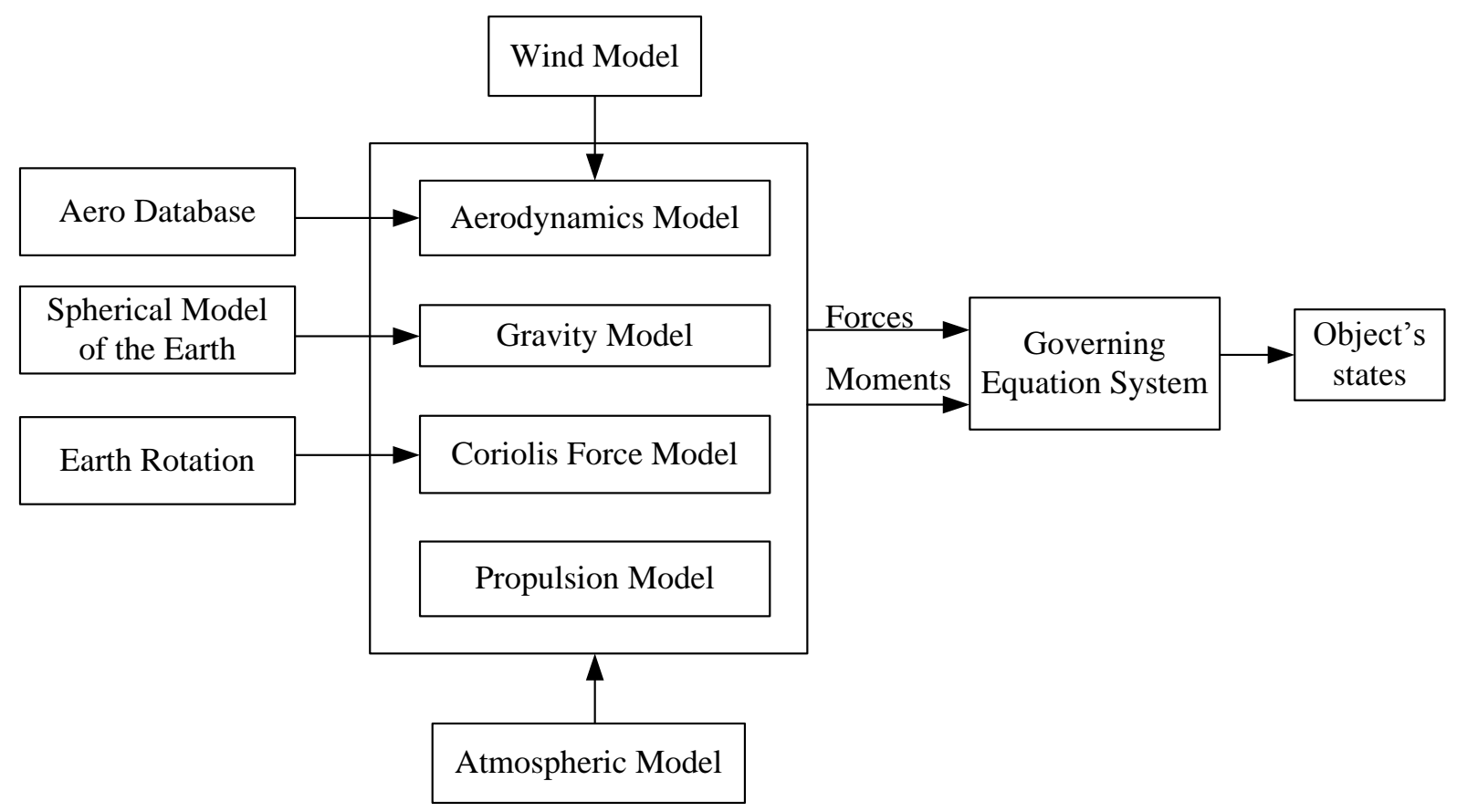

Figure 5: Dynamics model.

A non-linear Six Degree of Freedom (6DOF) model is built to describe the continuous states of an object in flight. It is dynamic behaviours, changing in time from releasing to hit the target, calculated by three main blocks as shown in Fig. 5.

The equations of motion are solved in Governing Equation System (GES) block. Six inputs of this block are three forces and three moments which respect to three main axes in Descartes coordinate system. The outputs are the current states of object. GES are contributed by applying Newton's laws and kinematic equation in several coordinate systems such as inertial frame, body frame, and wind frame. A certain coordinate system is selected for the convenience in express states equation follow reference $[2,3,15]$. The force $F=\left[F_{\mathrm{x}} F_{\mathrm{y}} F_{\mathrm{z}}\right]$ and moments $M=\left[M_{\mathrm{x}} M_{\mathrm{y}} M_{\mathrm{z}}\right]$ acting on the ballistic body are derived in Forces and Moments (FM) block including gravity model, Coriolis model, aerodynamics model and propulsion model (optional). The linear and angular positions are updated continuously and become inputs of FM block as the feedback. The environment block contains two main sub-blocks which are wind model and atmospheric model.

\subsection{Forces and moments}

Aerodynamics model: The block calculates all forces and moments due to the interaction between flight object and surrounding air. Basically, several state parameters are calculated such as angle of attack, side angle and velocity in order to determine six basic aerodynamic coefficients (lift, drag, side, pitch, roll and yaw) by looking-up the aerodynamic table which is built in Aero Database Generator representing forces and moments acting on flight object $[4,15]$.

Gravity model and Coriolis force model: Normally, the Earth's surface is assumed to be planar, the acceleration due to gravity is constant during the whole process. In fact, earth is considered as a spherical shape, gravity acceleration would be changed at different locations. In addition, the earth always rotates on its own axis; the flight object moving on the surface of earth would be affected by a force called Coriolis $[3,16]$. 
Propulsion model: Propulsion model depends on object of simulation. For example, bullet or bomb has no engine so that propulsion model is not enabled in this case. In other hand, in the case of rocket's trajectory simulation, the changing of mass and centre of gravity is considerable due to the existence of motor so that it should be included in calculation [17]. In this framework, propulsion model calculates propulsive force and moment for rocket during flying time. Besides, the model also creates an announcement for entire system (physical properties, aerodynamic model) about the mass reduction.

\subsection{Wind and atmospheric model}

Atmospheric model: The Earth's atmosphere includes several important parameters for trajectory simulation such as air density and speed of sound. Those variations respect to altitude, location, time of day, season, based on a standard atmosphere use in 1959 ARDC Model Atmosphere [17].

Wind model is given in Fig. 6.

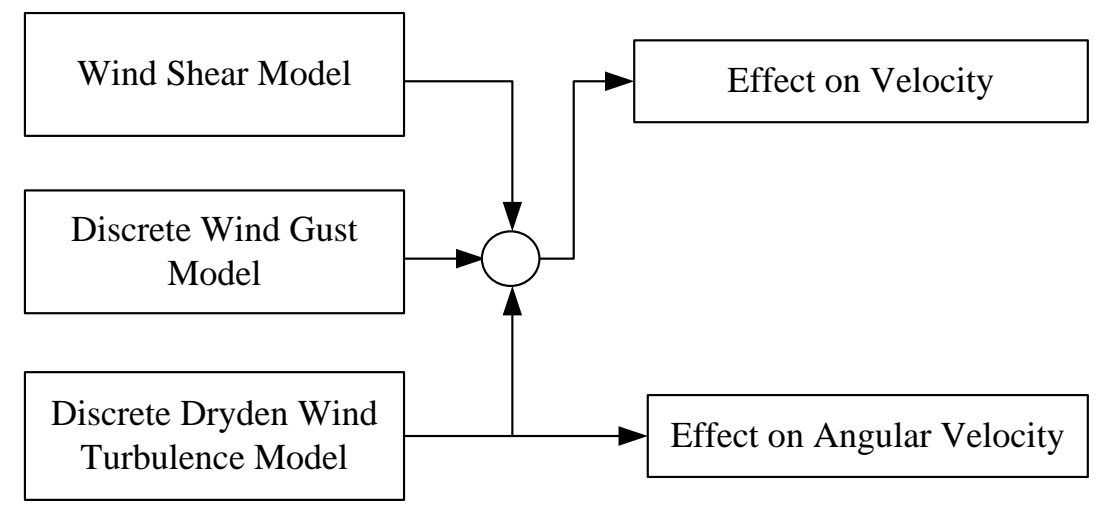

Figure 6: Wind model.

The effect of wind is approximated using mathematical model including two components: steady wind and turbulence wind. Wind longitudinal velocity $V_{\mathrm{w}}=\left[\begin{array}{lll}W_{\mathrm{x}} & W_{\mathrm{y}} & W_{\mathrm{z}}\end{array}\right]$ and wind angular velocity $W_{\mathrm{w}}=\left[\begin{array}{ll}W_{\mathrm{p}} & W_{\mathrm{q}} \\ W_{\mathrm{r}}\end{array}\right]$ impacting to the velocity and angular velocity of flight object are expressed in the body frame as shown in Fig. 6.

The output of wind model represents for effect of environment, is calculated and transferred to the aerodynamic model. Then, the total forces and moments are estimated which additionally includes gravitational model, Coriolis model and propulsion model. Figs. 7 and 8 point out the noise that is created by wind model acting on translational velocity and angular velocity.

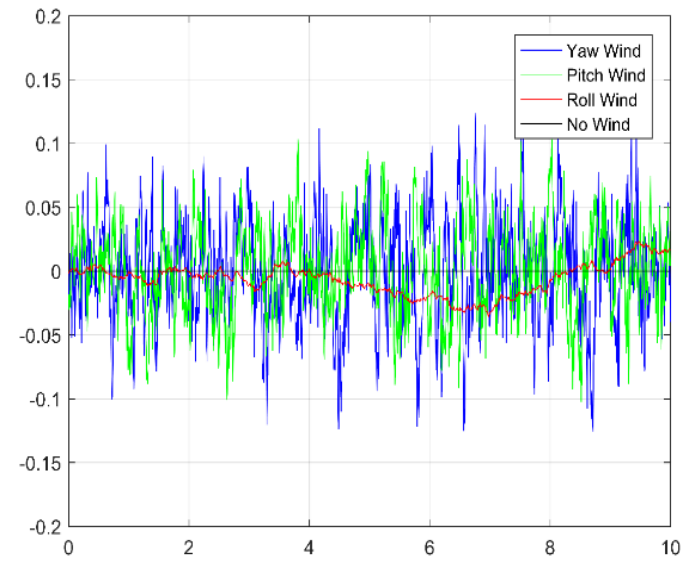

Figure 7: Noise of angular velocity.

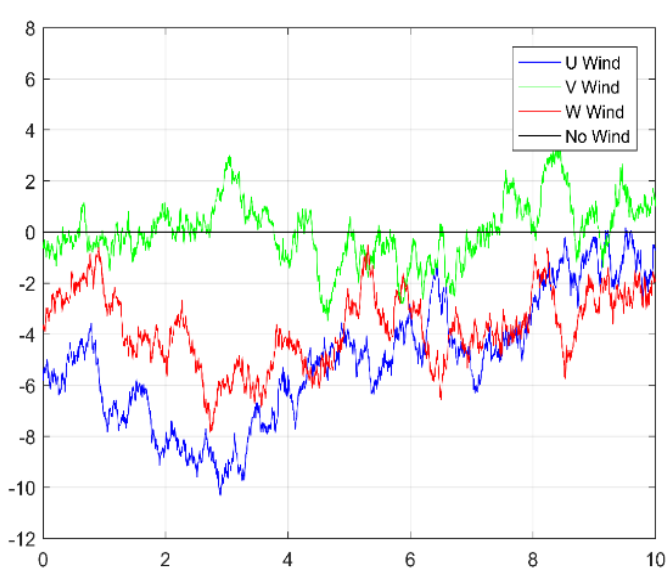

Figure 8: Noise of velocity. 


\section{VALIDATION AND VERIFICATION}

The bomb used in this validation is a typical fin-stabilized projectile with four fins as shown in Fig. 9. The physical properties included mass, diameter, pith, roll and yaw moment of inertia are $239.5 \mathrm{~kg}, 0.273 \mathrm{~m}, 2.35 \mathrm{kgm}^{2}, 56.74 \mathrm{kgm}^{2}, 56.74 \mathrm{kgm}^{2}$ respectively. In this case study, the object is normally released at attitude of 10000.0 feet with initial translational velocity of 200 and 400 knots in three different elevation angles 0, 10, 20 degrees and 0, 10, 30 degrees respectively. For the simplicity of study, the impact of environment is temporarily neglected. The comparison of trajectory between the current study and the study of Kasper and Wrenn [18] is shown in Figs. 10 and 11. The verification is carried out without wind effect.

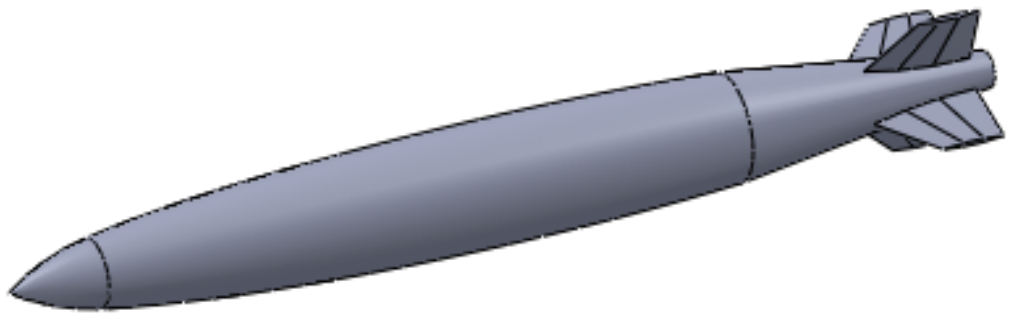

Figure 9: MK82 bomb.
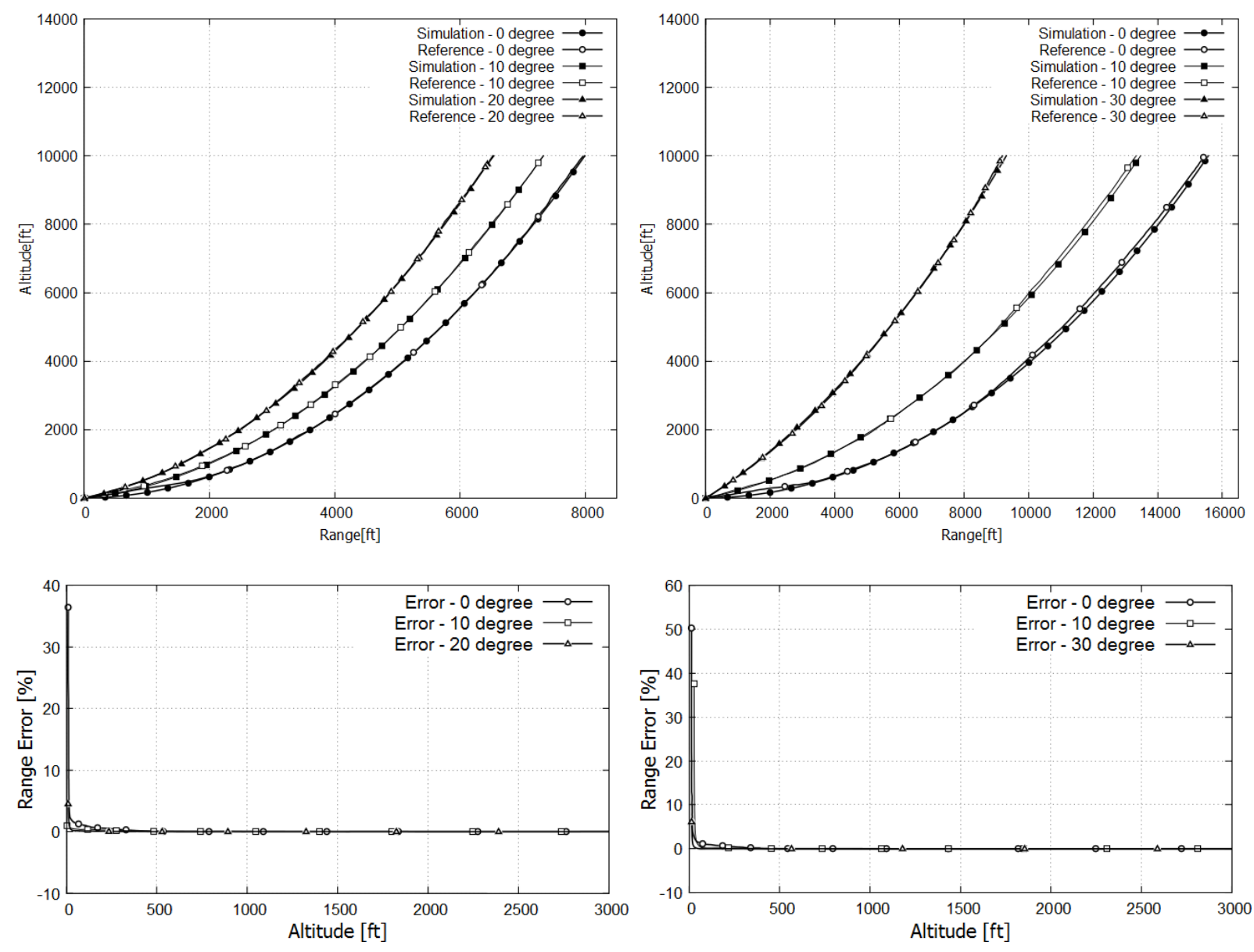

Figure 10: Altitude and range validated with reference at 200 knots.

Figure 11: Altitude and range validated with reference at 400 knots.

Figs. 10 and 11 show the comparisons between the trajectories that are generated from the current simulation and from the study of Kasper and Wrenn [18]. The result shows that 
current simulation is fairly similar to the reference. The difference between two studies is quite large at the beginning of simulation due to numerical error (which is still large comparing to range) and then the difference is smaller and smaller until the end of simulation $(<1 \%)$. The results show clearly that the mathematical model of simulation framework gives an exact solution in the case of altitude larger than 100 feet. The goal of use knots and feet as unit of velocity and length in this study is to unite current result with reference.

\section{RESULTS AND ANALYSIS}

To evaluate the effect of wind to the trajectory of the bomb, the initial chosen pitch angle is $30^{\circ}$. Figs. 12, 13, 14, and 15 represent the differentiations of trajectory in three cases: without wind effect, steady wind and turbulence wind.

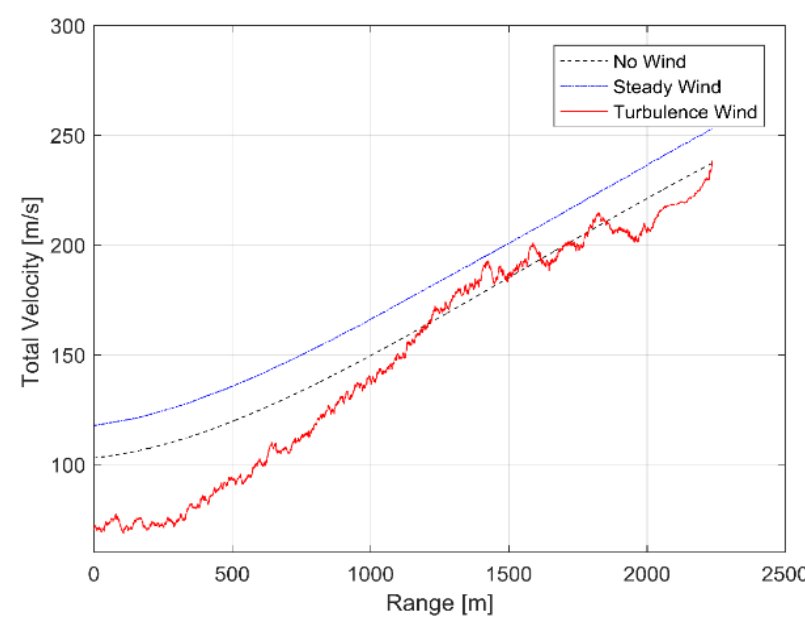

Figure 12: Comparison of total velocity.

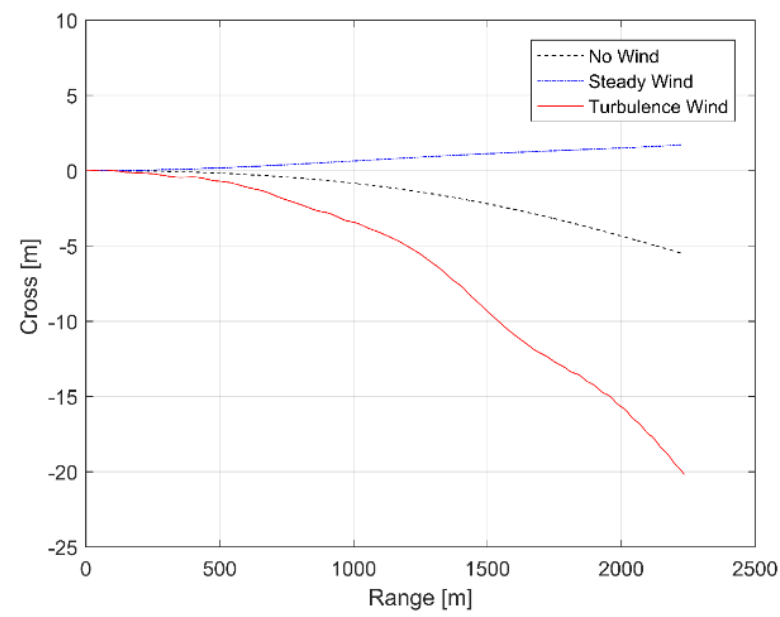

Figure 14: Comparison of side position.

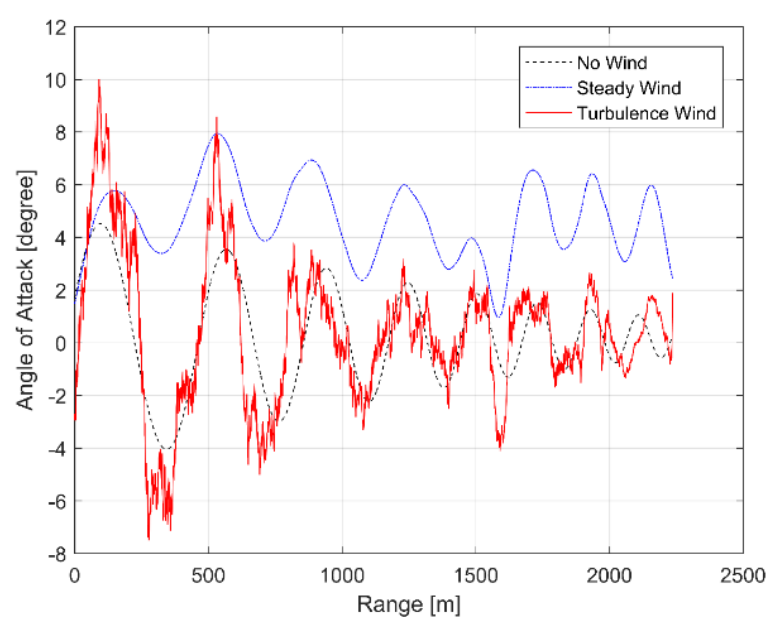

Figure 13: Comparison of angle of attack.

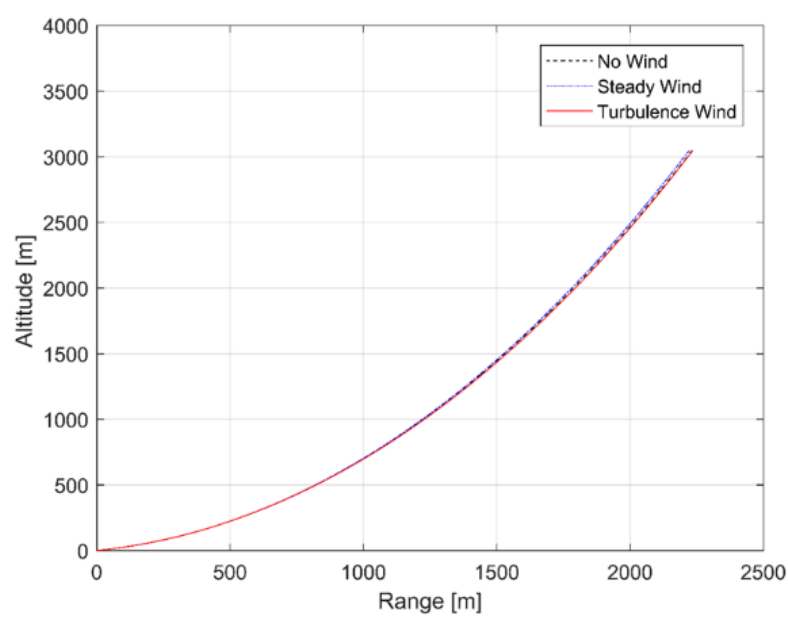

Figure 15: Comparison of dropping position.

Fig. 11 shows the change of total velocity during traveling time for each case study and Fig. 12 shows the change of angle of attack in respect to the traveling time. Both of figures indicate directly the impact of turbulence wind and gusty wind on the velocity and angle of the flight object. The behaviours of velocity and angle of attack are similar. However, the difference is small with the effect of static wind meanwhile the complex wind creates the significant difference with the intensity of the angle of attack. Besides, it could be seen the curves of velocity and angle of attack with the effect of steady wind are smooth and their changing is stable. In contrast, the curves with turbulence wind are more chaotic with 
component of high frequency because of the stochastic component acting on flight object body frame. The data from Fig. 14 show the effect of wind in cross range. At the initial moments, the difference is small. However, as time increases, the effects of turbulence wind and gust to projectile of the motion become larger. The deviation is approximately $6 \mathrm{~m}$ with the influence of the static wind, but it is up to $20 \mathrm{~m}$ with the effect of distortion. As shown in Figs. 14 and 15, the effect of wind in y-range is fairly larger than the one in x-range because of the aero-shape of bomb. Concretely, the effective area (where the aerodynamic forces act on the body) in the downward direction is quite smaller than in the side direction. The results show that the developed framework is able to predict the effect of environment into the trajectory of flight objects. By implementing this wind model, the program enables to reliably estimate the movement of object not only in short-range but also in long-range such as snipe shooting.

\section{CONCLUSIONS AND FUTURE WORK}

This research provides a framework to simulate the trajectory of ballistics under the effect of complex environment. Hence, the ballistic simulation is more and more close to reality and adequate for training demands. Based on the general geometry configuration, multi-fidelity analysis methods enable to economically generate the reliable aero-databases for fairly predicting trajectory of bomb, unguided missile and bullet as shown in results. The analyses have indicated that the effect of wind on the accuracy of projectiles is enormous, especially for the turbulence winds which is normally simplified in previous research. Evidently, the above results proved the efficiency and appropriation of proposed framework for the ballistic simulation. In future work, all the processes and mathematical model would be optimized and packaged with a control model to become a tool that estimate the missile launch applied to snipe shooting, smart weapon.

\section{ACKNOWLEDGEMENTS}

This research is fully funded by Viettel Institute of Research and Development which create the best environment to research the new technology.

\section{REFERENCES}

[1] Carlucci, D. E.; Jacobson, S. S. (2018). Ballistics: Theory and Design of Guns and Ammunition, $3^{\text {rd }}$ ed., CRC Press, Taylor \& Francis Group, Boca Raton

[2] Guan, J.; Yi, W. (2018). Modeling of dual-spinning projectile with canard and trajectory filtering, International Journal of Aerospace Engineering, Vol. 2018, Paper 1795158, 7 pages, doi:10.1155/2018/1795158

[3] Baranowski, L. (2013). Effect of the mathematical model and integration step on the accuracy of the results of computation of artillery projectile flight parameters, Bulletin of the Polish Academy of Sciences - Technical Sciences, Vol. 61, No. 2, 475-484, doi:10.2478/bpasts-2013-0047

[4] Baranowski, L. (2013). Equation of motion of a spin-stabilized projectile for flight stability testing, Journal of Theoretical and Applied Mechanics, Vol. 51, No. 1, 235-246

[5] Zyluk, A. (2014). Numerical simulation of the effect of wind on the missile motion, Journal of Theoretical and Applied Mechanics, Vol. 52, No. 2, 335-344

[6] Gkritzapis, D. N.; Panagiotopoulos, E. E.; Margaris, D. P.; Papanikas, D. G. (2007). Computational atmospheric trajectory simulation analysis of spin-stabilized projectiles and small bullets, AIAA Atmospheric Flight Mechanics Conference and Exhibit, Paper 2007-6584, doi:10.2514/6.2007-6584

[7] Hainz, L. C.; Costello, M. (2005). Modified projectile linear theory for rapid trajectory prediction, Journal of Guidance, Control and Dynamics, Vol. 28, No. 5, 1006-1014, doi: $10.2514 / 1.8027$ 
[8] McDonnell Douglas Astronautics Company, St. Louis (1979) \& Public Domain Aeronautical Software - update (1999). The USAF Stability and Control Datcom, Santa Cruz

[9] Kostić, Č. (2015). Review of the Spalart-Allmaras turbulence model and its modifications to three-dimensional supersonic configurations, Scientific Technical Review, Vol. 65, No. 1, 43-49, doi:10.5937/STR1501043K

[10] Ghoreyshi, M.; Badcock, K. J.; Woodgate, M. A. (2008). Integration of multi-fidelity methods for generating an aerodynamic model for flight simulations, $46^{\text {th }}$ AIAA Aerospace Sciences Meeting and Exhibit, Paper 2008-197, 11 pages, doi:10.2514/6.2008-197

[11] He, L.; Zhou, Y.; Qian, W.-Q.; Wang, Q. (2017). Aerodynamic data fusion with a multi-fidelity surrogate modeling method, $7^{\text {th }}$ European Conference for Aeronautics and Space Sciences (EUCASS), 10 pages, doi:10.13009/EUCASS2017-108

[12] Zhang, Q. (2017). Development of a data fusion framework for the aerodynamic analysis of launchers, Master Thesis, Delft University of Technology, Delft

[13] Lophaven, S. N.; Nielsen, H. B.; Søndergaard, J. (2002). DACE - A Matlab Kriging Toolbox, IMM, Technical University of Denmark, Kgs. Lyngby

[14] Couckuyt, I.; Dhaene, T.; Demeester, P. (2013). ooDACE Toolbox, A Matlab Kriging toolbox: Getting started, Department of Information Technology, Ghent University, Ghent

[15] Beard, R. W.; McLain, T. W. (2012). Small Unmanned Aircraft - Theory and Practice, Princeton University Press, Princeton

[16] NATO (2009). STANAG 4355 - The modified point mass and five degrees of freedom trajectory models ( $3^{\text {rd }}$ ed.), Brussels

[17] Siouris, G. M. (2004). Missile Guidance and Control Systems, Springer-Verlag, New York

[18] Kasper, M.; Wrenn, A. (1966). MK81 and MK82 bomb release curves, United States Naval Ordnance Laboratory, White Oak 